\title{
NOTE ON THE GLOBAL DIMENSION OF A CERTAIN RING
}

\section{GEORGE S. RINEHART ${ }^{1}$}

If $M$ is a module over a ring $S$, we denote by $d_{S}(M)$ the projective dimension of $M$ as an $S$-module, and by gl.d.( $S$ ) the global dimension of $S$; i.e., the sup of $d_{S}(M)$ as $M$ runs over all left $S$-modules [2, VI].

Let $S$ be a commutative ring with identity element 1 , and let $F$ be a subring of $S$ containing 1 . Let $T$ be the left $R$-module of all $F$-derivations of $S$. $T$ may be regarded as a Lie algebra over $F$ in the natural fashion. Regarding $S$ as an abelian Lie algebra over $F$, we construct a Lie algebra $L$ over $F$, containing $S$ as an ideal and $T$ as a subalgebra, by letting $L$ be the semidirect sum $S+T$, the commutation being given by

$$
\left[s_{1}+t_{1}, s_{2}+t_{2}\right]=\left(t_{1}\left(s_{2}\right)-t_{2}\left(s_{1}\right)\right)+\left[t_{1}, t_{2}\right] .
$$

Let $U$ denote the universal enveloping algebra of $L$, and denote the canonical injection of $L$ into $U$ by $x \rightarrow x^{\prime}$. Let $I$ be the ideal of $L^{\prime} U$ generated by the elements $r^{\prime} x^{\prime}-(r x)^{\prime}$, with $r$ in $S$ and $x$ in $L$. Put $V=L^{\prime} U / I$. To each element of $V$ there corresponds a differential operator on $R$ in the natural fashion, and $S$ thus becomes a $V$-module. The $F$-algebra $V$ has been used by Hochschild, Kostant and Rosenberg for the theory of the differential forms on $S$. They have shown [4] that if $F$ is a perfect field and $S$ is a regular affine $F$-algebra of Krull dimension $n, d_{V}(S)=n$ and gl.d. $(V) \leqq 2 n$.

We are chiefly concerned here with the problem of the precise determination of the global dimension of $V$ in the simplest nontrivial case, where $S=F\left[y_{1}, \cdots, y_{n}\right]$ is the polynomial algebra in $n$ variables over an arbitrary field $F$. In this case, $T$ is a free $S$-module on $n$ generators, the partial differentiations with respect to the variables. From this it is easily seen that $V$ may be identified with $\left(S \otimes_{F} S\right) / J, J$ being the ideal generated by the elements $y_{i} \otimes y_{j}-y_{j} \otimes y_{i}-\delta_{i j} 1 \otimes 1$, where $\delta_{i j}$ is the Kronecker delta. Writing $x_{i}$ for $y_{i} \otimes 1$ and $y_{i}$ for $1 \otimes y_{i}$, we have $V=R_{n}=F\left[y_{1}, \cdots, y_{n}\right]\left\{x_{1}, \cdots, x_{n}\right\}$, with $x_{i} x_{j}=x_{j} x_{i}$ and $x_{i} y_{j}-y_{j} x_{i}=\delta_{i j}$.

From the above, we have $n \leqq$ gl.d. $\left(R_{n}\right) \leqq 2 n$. If $F$ has characteristic $\neq 0$, we easily show gl.d. $\left(R_{n}\right)=2 n$. The case for characteristic zero turns out to be hard. Here we obtain a definite result only for $n=1$,

Received by the editors May 1, 1961.

1 The author is a National Science Foundation Fellow. This work was done under the direction of Professor G. P. Hochschild, for whose advice and encouragement the author is most grateful. 
showing that gl.d. $\left(R_{1}\right)=1$. This result in turn gives a slight strengthening of the above inequality $\left(n \leqq\right.$ gl.d. $\left(R_{n}\right) \leqq 2 n-1$, if $F$ has characteristic 0 ).

The considerations here are independent of the Hochschild-KostantRosenberg result.

1. By a theorem of M. Auslander [1, Theorem 1], we know that gl.d. $(S) \leqq 1+\sup d_{S}(I)$, as $I$ runs through all left ideals of $S$. We therefore begin with some considerations of the ideal structure of the ring $R=F[y]\{x\}$ (with $x y-y x=1$ ).

Every element of $R$ can be written uniquely in the form

$$
\theta=\sum_{i=0}^{k} f_{i}(y) x^{i}
$$

where each $f_{i}(y)$ is an element of $F[y]$. If $f_{k}(y) \neq 0$, we say that $\theta$ is of degree $k$ (written $\delta^{0}(\theta)=k$ ) and has leading coefficient $f_{k}(y)$. We have

$$
x^{n} f(y)=\sum_{k=0}^{n}\left(\begin{array}{l}
n \\
k
\end{array}\right) f^{(k)}(y) x^{n-k}
$$

It follows from this that $\delta^{0}(\theta \phi)=\delta^{0}(\theta)+\delta^{0}(\phi)$, for all $\theta$ and $\phi$ in $R$.

Let $I$ be any (left) ideal of $R$ and let $I_{k} \subset F[y]$ be the ideal consisting of the leading coefficients of those elements of $I$ which have degree $k$. Let $g_{k}(y)$ be the monic polynomial that generates $I_{k}$ as an ideal in $F[y]$. Let $\theta_{k}$ be an element of $I$ of degree $k$ with leading coefficient $g_{k}(y)$. Then if $\phi \in I$ has degree $s, \phi-h(y) \theta_{s}$ has degree less than $s$, for some $h(y) \in F[y]$. Hence, by an induction on the degree,

$$
I=\sum_{k=0}^{\infty} F[y] \theta_{k} .
$$

Now, since

$$
x \sum_{i=0}^{k} f_{i}(y) x^{i}=f_{k}(y) x^{k+1}+\sum_{i=0}^{k}\left(f_{i}^{\prime}(y)+f_{i-1}(y)\right) x^{i},
$$

we have $I_{k} \subset I_{k+1}$. Hence $g_{k+1}(y)$ divides $g_{k}(y)$. Therefore, for some sufficiently large $m, g_{k}(y)=g_{m}(y)$ for every $k \geqq m$. Hence, by (4), we can choose $\theta_{k}=x^{k-m} \theta_{m}$, for every $k \geqq m$. Then, by (3),

$$
I=\sum_{k=0}^{m} R \theta_{k} .
$$

Let $d_{k}(y)=g_{k}(y) / g_{k+1}(y)$. Then $\delta^{0}\left(d_{k}(y) \theta_{k+1}-x \theta_{k}\right) \leqq k$, by (4), so that 


$$
d_{k}(y) \theta_{k+1} \in \sum_{j=0}^{k} F[y] \theta_{j} \subset \sum_{j=0}^{k} R \theta_{j}
$$

REMARK. $R$ is not a principal ideal domain.

Consider $I=R x^{2}+R(y x-1)$. Note that $x(y x-1)=y x^{2}$. Hence $I=R x^{2}+F[y](y x-1)$. Using (1), it is easy to see that $I \cap F[y]=(0)$. Now suppose $I=R \phi$. Then $y x-1=\phi_{1} \phi$ and, using the remark following (2), we conclude that $\delta^{0}(\phi)=s \leqq 1$ and $\delta^{0}\left(\phi_{1}\right)=1-s$. Since $I \cap F[y]$ $=(0)$, we must have $s>0$, whence $s=1$ and $\phi_{1} \in F[y]$. From this it follows that actually $\phi_{1} \in F$, so that $I=R(y x-1)$. Since (2) shows that $x^{2} \notin R(y x-1)$, this is a contradiction.

LEMMA 1. Let $F$ have characteristic 0 and let $I$ be a proper left (right) ideal in $R$ containing a monic irreducible polynomial $p(y) \in F[y]$. Then $I=R p(y)(I=p(y) R)$.

Proof. Write $I$ as in (5) with $m$ minimal. Clearly $\theta_{0}=p(y)$, and it suffices to show that $m=0$. Suppose not. Then $g_{k}(y) \neq g_{0}(y)=p(y)$, for some $k$. Choose a minimal such $k$. Since $g_{k}(y)$ divides $p(y), g_{k}(y)$ $=1$. Hence $\theta_{k}=x^{k}+\sum_{i=0}^{k-1} f_{i}(y) x^{i}$. Hence

$$
p(y) \theta_{k}-x^{k} p(y)=\sum_{i=0}^{k-1}\left(p(y) f_{i}(y)-\left(\begin{array}{l}
k \\
i
\end{array}\right) p^{(k-i)}(y)\right) x^{i} .
$$

Since this element is in $I, g_{k-1}(y)$ divides its leading coefficient. But $g_{k-1}(y)=p(y)$. Hence $p(y)$ divides $p(y) f_{k-1}(y)-k p^{\prime}(y)$, whence $p(y)$ $=1$, a contradiction.

A similar proof gives the result for right ideals.

2. Now we turn to the question of the global dimension of $R_{n}$.

Proposition 1. Let $R^{*}$ be the opposite algebra of $R$. Then $d_{R \otimes R^{*}}(R)$ $=2$.

Proof. As an $R \otimes R^{*}$-module, $R$ is isomorphic to $R \otimes R^{*} / K$, where $K$ is the ideal generated by $x \otimes 1-1 \otimes x^{*}$ and $y \otimes 1-1 \otimes y^{*}$. It is suffi-

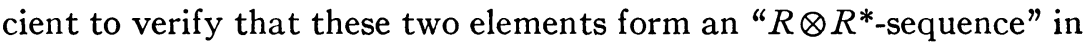
the sense that they commute with each other and that the only left multiples of one lying in the left ideal generated by the other are multiples by an element in that ideal; for then a standard resolution due to Koszul shows that $d_{R \otimes R^{*}}(R)=2$ [2, VIII, 4.2]. But we can define an $F$-isomorphism between $R_{2}$ and $R \otimes R^{*}$ by $x_{1} \rightarrow x \otimes 1-1 \otimes x^{*}$, $x_{2} \rightarrow 1 \otimes x^{*}, y_{1} \rightarrow y \otimes 1, y_{2} \rightarrow y \otimes 1-1 \otimes y^{*}$, and it is easily seen that $x_{1}$ and $y_{2}$ form an " $R_{2}$-sequence."

CoROllaRY. gl.d. $\left(R_{n}\right) \leqq g l . d .(R)+2(n-1)$; and $g l . d .\left(R_{n}\right) \leqq 2 n$. 
Proof. For $n>1, R_{n}=R_{n-1} \otimes_{F} R$. By [3, Prop. 2], the global dimension of the latter does not exceed gl.d. $\left(R_{n-1}\right)+d_{R \otimes R^{*}}(R)$ $=$ gl.d. $\left(R_{n-1}\right)+2$. Hence, by iteration, the first result is obtained. The second follows from it by one more iteration, since $R=F \otimes_{F} R$, and gl.d. $(F)=0$.

Proposition 2. If $F$ has characteristic zero, gl.d.(R) $\leqq 1$.

Proof. Let $I$ be an ideal of $R$, written as in (5). For $\theta_{k} \neq 0$, choose $d_{k, 0}(y), d_{k, 1}(y), \cdots, d_{k, q_{k}}(y)$ in $F[y]$ such that $d_{k, 0}(y)=d_{k}(y)$, $d_{k, q_{k}}(y)=1, d_{k, j+1}(y)$ divides $d_{k, j}(y)$, and $d_{k, j}(y) / d_{k, j+1}(y)$ is an irreducible polynomial. Let $k_{0}$ be minimal such that $\theta_{k_{0}} \neq 0$. Set $\phi_{0}=\theta_{k_{0}}$, $\phi_{1}=d_{k_{0}, 1}(y) \theta_{k_{0}+1}, \cdots, \quad \phi_{q_{k_{0}}}=d_{k_{0}, q_{k_{0}}}(y) \theta_{k_{0}+1}=\theta_{k_{0}+1}, \quad \phi_{q_{k}+1}$ $=d_{k_{0}+1,1}(y) \theta_{k_{0}+2}, \cdots$ etc. Let $J_{k}^{0}=\sum_{j=0}^{k} R \phi_{j}$. Then, using (6),

$$
p_{k}(y) \phi_{k+1} \in J_{k}
$$

for some irreducible polynomial $p_{k}(y) \in F[y]$; and $J_{t}=I$ for $t=\sum_{k=k_{0}}^{m-1} q_{k}$.

Now $J_{0}$ is $R$-free and hence $d_{R}\left(J_{0}\right)=0$. Assume inductively that $d_{R}\left(J_{k}\right)=0$. We will show $d_{R}\left(J_{k+1}\right)=0$, and conclude by induction that $d_{R}(I)=0$, and hence that gl.d. $(R) \leqq 1$. We assume that $\phi_{k+1}$ is not in $J_{k}$, since otherwise there is nothing to prove.

Lemma 2. The restriction map $\rho: \operatorname{Hom}_{R}\left(J_{k+1}, R\right) \rightarrow \operatorname{Hom}_{R}\left(J_{k}, R\right)$ is not surjective.

Proof. Since $J_{k}$ is finitely generated, there is a finitely generated free $R$-module $E$ and an epimorphism $p: E \rightarrow J_{k}$. Since by inductive hypothesis $J_{k}$ is projective, there is a homomorphism $i: J_{k} \rightarrow E$ such that $p \circ i$ is the identity. Consider the commutative diagram

$$
\begin{gathered}
\operatorname{Hom}\left(J_{k+1}, E\right) \stackrel{\rho_{1}}{\rightarrow} \operatorname{Hom}\left(J_{k}, E\right) \\
\downarrow \\
\operatorname{Hom}\left(J_{k+1}, J_{k}\right) \stackrel{\rho_{2}}{\rightarrow} \operatorname{Hom}\left(J_{k}, J_{k}\right)
\end{gathered}
$$

where the vertical maps are induced by $p$. If $\rho$ were surjective so would be $\rho_{1}$, by a direct sum argument. Then $i$ would be in the image of $\rho_{1}$, so that the identity would be in the image of $\rho_{2}$. But if $\rho_{2}(a)$ were the identity, for some $a \in \operatorname{Hom}\left(J_{k+1}, J_{k}\right)$, we would have $a\left(p_{k}(y) \phi_{k+1}\right)=p_{k}(y) \phi_{k+1}$ by (7). Then $p_{k}(y) a\left(\phi_{k+1}\right)=p_{k}(y) \phi_{k+1}$, whence $a\left(\phi_{k+1}\right)=\phi_{k+1}$, whence $\phi_{k+1} \in J_{k}$, contrary to assumption. Thus the lemma is proved.

Now $J_{k+1} / J_{k} \cong R \phi_{k+1} /\left(R \phi_{k+1} \cap J_{k}\right)$. Hence there is an $R$-epimorphism $\beta: R \rightarrow J_{k+1} / J_{k}$ such that $\beta(1)=\phi_{k+1}+J_{k}$. Since $\beta\left(p_{k}(y)\right)=0$ by 
(7), $\beta$ induces an isomorphism between $R / R p_{k}(y)$ and $J_{k+1} / J_{k}$, by Lemma 1. Thus we have an exact sequence

$$
(0) \rightarrow J_{k} \rightarrow J_{k+1} \rightarrow R / R p_{k}(y) \rightarrow(0),
$$

from which we obtain the exact sequence

$$
\begin{aligned}
\operatorname{Hom}\left(J_{k+1}, R\right) & \stackrel{\rho}{\rightarrow} \operatorname{Hom}\left(J_{k}, R\right) \stackrel{\gamma}{\rightarrow} \operatorname{Ext}^{1}\left(R / R p_{k}(y), R\right) \stackrel{\delta}{\rightarrow} \operatorname{Ext}^{1}\left(J_{k+1}, R\right) \\
& \rightarrow \operatorname{Ext}^{1}\left(J_{k}, R\right)=(0) .
\end{aligned}
$$

The right $R$-module structure of $R$ induces a right $R$-module structure on the groups of (8), and the maps are then $R$-homomorphisms. From the projective resolution

$$
(0) \rightarrow R p_{k}(y) \rightarrow R \rightarrow R / R p_{k}(y) \rightarrow(0)
$$

we compute $\operatorname{Ext}^{1}\left(R / R p_{k}(y), R\right)=R / p_{k}(y) R$. By Lemma 1, this is simple as a right $R$-module. But, by Lemma 2 , the map $\rho$ in (8) is not surjective, and therefore $\gamma \neq 0$. Hence $\gamma$ must be surjective, and therefore $\delta=0$. Since $\delta$ is surjective,

$$
\operatorname{Ext}^{1}\left(J_{k+1}, R\right)=(0) \text {. }
$$

Since gl.d. $(R) \leqq 2$, by the previous corollary,

$$
d_{R}\left(J_{k+1}\right) \leqq 1
$$

so that there is a projective resolution

$$
(0) \rightarrow X_{1} \rightarrow X_{0} \rightarrow J_{k+1} \rightarrow(0)
$$

of $J_{k+1}$. Since $R$ is noetherian, $X_{0}$ and $X_{1}$ can be chosen to be finitely generated. Let $E$ be $R$-free. Then since by (9) the map $\operatorname{Hom}\left(X_{0}, R\right)$ $\rightarrow \operatorname{Hom}\left(X_{1}, R\right)$ is surjective, it is easily seen that $\operatorname{Hom}\left(X_{0}, E\right)$ $\rightarrow \operatorname{Hom}\left(X_{1}, E\right)$ is also surjective; i.e.,

$$
\operatorname{Ext}^{1}\left(J_{k+1}, E\right)=(0) \text {. }
$$

Now let $C$ be any $R$-module, and consider an exact sequence

$$
(0) \rightarrow M \rightarrow E \rightarrow C \rightarrow(0)
$$

with $E R$-free. We obtain an exact sequence

$$
\operatorname{Ext}^{1}\left(J_{k+1}, E\right) \rightarrow \operatorname{Ext}^{1}\left(J_{k+1}, C\right) \rightarrow \operatorname{Ext}^{2}\left(J_{k+1}, M\right) .
$$

Using (10) and (11), we conclude $\operatorname{Ext}^{1}\left(J_{k+1}, C\right)=(0)$. Hence $d_{R}\left(J_{k+1}\right)$ $=0$. This completes the proof of Proposition 2 .

THEOREM. If $F$ has characteristic $\neq 0$, gl.d. $\left(R_{n}\right)=2 n$. If $F$ has characteristic $0, n \leqq g l . d .\left(R_{n}\right) \leqq 2 n-1$. 
Proof. $x_{1}, \cdots, x_{n}$ is an " $R_{n}$-sequence." (See the proof of Proposition 1.) Hence the module obtained by factoring out the ideal generated by $x_{1}, \cdots, x_{n}$ has dimension $n$. If $F$ has characteristic $p \neq 0$, $x_{1}, \cdots, x_{n}, y_{1}^{p}, \cdots, y_{n}^{p}$ is such a sequence, and hence determines a module of dimension $2 n$. In the light of Proposition 2 and the corollary to Proposition 1, this completes the proof of the theorem.

\section{BiBLIOGRAPHY}

1. M. Auslander, On the dimension of modules and algebras. III, Nagoya Math. J. 9 (1955), 67-77.

2. H. Cartan and S. Eilenberg, Homological algebra, Princeton Univ. Press, Princeton, N. J., 1956.

3. S. Eilenberg, A. Rosenberg and D. Zelinsky, On the dimension of modules and algebras. VIII, Nagoya Math. J. 12 (1957), 71-93.

4. G. Hochschild, B. Kostant and A. Rosenberg, Differential forms on regular affine algebras, Trans. Amer. Math. Soc. 102 (1962), 383-408.

University of California, Berkeley 